\title{
Identification and expression analysis of the GDSL esterase/lipase family genes, and the characterization of SaGLIP8 in Sedum alfredii Hance under cadmium stress
}

\author{
He Li ${ }^{\text {Equal first author, 1, 2, } 3}$, Xiaojiao Han ${ }^{\text {Equal first author, 2, } 3}$, Wenmin Qiu ${ }^{2,3}$, Dong Xu ${ }^{2,3}$, Ying Wang ${ }^{2,3}$, Miao Yu ${ }^{2,3}$, Xianqi Hu \\ Corresp., 1 , Renying Zhuo ${ }^{\text {Corresp. 2, } 3}$ \\ ${ }^{1}$ College of Plant Protection, Yunnan Agricultural University, Kunming, Yunnan, China \\ State Key Laboratory of Tree Genetics and Breeding, Chinese Academy of Forestry, Beijing, China \\ ${ }^{3}$ Key Laboratory of Tree Breeding of Zhejiang Province, The Research Institute of Subtropical of Forestry, Chinese Academy of Forestry, Hangzhou, \\ Zhejiang, China
}

Corresponding Authors: Xianqi Hu, Renying Zhuo

Email address: xqh@ynau.edu.cn, zhuory@gmail.com

Background: The herb Sedum alfredii (S. alfredii) Hance is a hyper-accumulator of heavy metals [cadmium (Cd), zinc $(\mathrm{Zn})$ and lead $(\mathrm{Pb})$ ]; therefore, it could be a candidate plant for efficient phytoremediation. The GDSL esterase/lipase protein (GELP) family plays important roles in plant defense and growth. Although the GELP family members in a variety of plants have been cloned and analyzed, there are limited studies on the family's responses to heavy metal-stress conditions.

Methods: Multiple sequence alignments and phylogenetic analyses were performed according to the criteria described. A WGCNA was used to construct co-expression regulatory networks. The roots of $S$. alfredii seedlings were treated with $100 \mu \mathrm{M} \mathrm{CdCl}_{2}$ for qRT-PCR to analyze expression levels in different tissues. SaGLIP8 was transformed into the $C d$ sensitive mutant strain yeast $\Delta y c f 1$ to investigate its role in resistance and accumulation to $\mathrm{Cd}$.

Results: We analyzed GELP family members from genomic data of $S$. alfredii. A phylogenetic tree divided the 80 identified family members into three clades. The promoters of the 80 genes contained certain elements related to abiotic stress, such as TC-rich repeats (defense and stress responsiveness), heat shock elements (heat stress) and MYB-binding sites (drought-inducibility). In addition, 66 members had tissue-specific expression patterns and significant responses to Cd stress. In total, 13 hub genes were obtained, based on an existing S. alfredii transcriptome database, that control 459 edge genes, which were classified into five classes of functions in a co-expression subnetwork: cell wall and defense function, lipid and esterase, stress and tolerance, transport and transcription factor activity. Among the hub genes, Sa13F.102 (SaGLIP8), with a high expression level in all tissues, could increase Cd tolerance and accumulation in yeast when overexressed.

Conclusion: Based on genomic data of $S$. alfredii, we conducted phylogenetic analyses, as well as conserved domain, motif and expression profiling of the GELP family under Cd-stress conditions. SaGLIP8 could increase $\mathrm{Cd}$ tolerance and accumulation in yeast. These results indicated the roles of GELPs in plant responses to heavy metal exposure and provides a theoretical basis for further studies of the SaGELP family's functions. 


\section{Identification and expression analysis of the GDSL}

2 esterase/lipase family genes, and the characterization of SaGLIP8

$4 \mathrm{He} \mathrm{Li}^{1}$, 2, 3\#, Xiaojiao Han², 3\#, Wenmin Qiü, 3, Dong $\mathrm{Xu}^{2,3}$, Ying Wang 2, 3, Miao $\mathrm{Yu}^{2,3}$, Xianqi Hu1*,

5 Renying Zhuo $2,3^{*}$

$6 \quad{ }^{1}$ College of Plant Protection, Yunnan Agricultural University, Kunming Yunnan, China

$7 \quad 2$ State Key Laboratory of Tree Genetics and Breeding, Chinese Academy of Forestry, Beijing, China

$8{ }^{3}$ Key Laboratory of Tree Breeding of Zhejiang Province, The Research Institute of Subtropical

9 Forestry, Chinese Academy of Forestry, Hangzhou Zhejiang, China

$10 *$ Corresponding author:

11 Xianqi $\mathrm{Hu}^{1}$

12 Fengyuan road 452\#, Kunming, Yunnan, 650201, China

13 Email address: xqhoo@126.com

14 Renying Zhuo 2,3

15 Daqiao road 73\#, Fuyang, Hangzhou, Zhejiang, 311400, China

16 Email address: zhuory@gmail.com

\# These authors contributed equally to the work. 


\section{Abstract}

Background: The herb Sedum alfredii ( $S$. alfredii) Hance is a hyper-accumulator of heavy metals [cadmium $(\mathrm{Cd})$, zinc $(\mathrm{Zn})$ and lead $(\mathrm{Pb})]$; therefore, it could be a candidate plant for efficient phytoremediation. The GDSL esterase/lipase protein (GELP) family plays important roles in plant defense and growth. Although the GELP family members in a variety of plants have been cloned and analyzed, there are limited studies on the family's responses to heavy metal-stress conditions.

Methods: Multiple sequence alignments and phylogenetic analyses were performed according to the criteria described. A WGCNA was used to construct co-expression regulatory networks. The roots of $S$. alfredii seedlings were treated with $100 \mu \mathrm{M} \mathrm{CdCl}$ for qRT-PCR to analyze expression levels in different tissues. SaGLIP8 was transformed into the Cd sensitive mutant strain yeast $\Delta y c f 1$ to investigate its role in resistance and accumulation to $\mathrm{Cd}$.

Results: We analyzed GELP family members from genomic data of $S$. alfredii. A phylogenetic tree divided the 80 identified family members into three clades. The promoters of the 80 genes contained certain elements related to abiotic stress, such as TC-rich repeats (defense and stress responsiveness), heat shock elements (heat stress) and MYB-binding sites (drought-inducibility). In addition, 66 members had tissue-specific expression patterns and significant responses to Cd stress. In total, thirteen hub genes were obtained, based on an existing $S$. alfredii transcriptome database, that control 459 edge genes, which were classified into five classes of functions in a co-expression subnetwork: cell wall and defense function, lipid and esterase, stress and tolerance, transport and transcription factor activity. Among the hub genes, Sa13F.102 (SaGLIP8), with a high expression level in all tissues, could increase Cd tolerance and accumulation in yeast when overexressed. 
43 Conclusion: Based on genomic data of S. alfredii, we conducted phylogenetic analyses, as well as

44 conserved domain, motif and expression profiling of the GELP family under Cd-stress conditions. SaGLIP8 could increase Cd tolerance and accumulation in yeast. Our results revealed that the SaGELP family responds to cadmium stress. These results indicated the roles of GELPs in plant responses to heavy metal exposure and provides a theoretical basis for further studies of the SaGELP family's functions.

\section{Introduction}

Cadmium (Cd) is an important environmental pollutant and inorganic toxicant, which has serious impacts

on the growth and development of organisms (Liu et al. 2014). Cd has a wide range of sources, including

electroplating, coatings and mining (Satarug et al. 2011), and can enter organisms through water and food, resulting in adverse effects (Nair et al. 2013). In mammals, Cd can cause a variety of diseases such as renal failure and blood pressure disorders, and it can also lead to osteoporosis, diabetes and neurological disorders (Jarup \& Akesson 2009; Messner \& Bernhard 2010). Cd accumulation in plants affects water inhibition (Oono et al. 2014). Heavy metal-contaminated soil threatens agriculture and food safety (Zhu et al. 2018). Some hyperaccumulative plants adapted to metalliferous soils in different ways (Gao et al. 2014). Hyperaccumulators, for instance, Cd/zinc $(\mathrm{Zn}) /$ lead $(\mathrm{Pb})$ co-hyperaccumulator Sedum alfredii Hance, arsenic (As) and $\mathrm{Pb}$ co-hyperaccumulator Eremochloa ciliaris (Linn.) Merr. and manganese (Mn) hyperaccumulator Phytolacca acinosa Roxb are able to accumulate heavy metals in aboveground tissues but not exhibit symptoms of poisoning, and are widely used in phytoremediation (Pilon-Smits 2005). 
63 The hyperaccumulating ecotype $\mathrm{S}$. alfredii Hance, with a high tolerance to $\mathrm{Zn}, \mathrm{Cd}$ and $\mathrm{Pb}$, can grow

64 normally in soil having Cd concentrations up to $400 \mathrm{mg} \cdot \mathrm{kg}^{-1}$ (Tian et al. 2017; Xing et al. 2013). Leaf

65 vacuolar isolation is currently considered to be the main mechanism of Cd detoxification in

66 hyperaccumulator plants (Rascio \& Navari-Izzo 2011; Sharma et al. 2016). Genes related to Cd transport

67 (Rascio \& Navari-Izzo 2011), chelation (Zhang et al. 2011) and reactive oxygen species (ROS) scavenging

68 (Li et al. 2017) have been identified. SpHMA3 (Heavy metal ATPase 3) from Sedum plumbizincicola

69 overexpressing in the non-hyperaccumulating ecotype of $S$. alfredii greatly increased its tolerance to, and

70 cadmium detoxification is achieved by chelation of toxic or excessive heavy metals into the vacuole. (Liu et

71 al. 2017). In addition, SaCAX2 (cation exchanger 2), SaMT2 (metallothionein 2) and SaCulZn SOD

72 (superoxide dismutase) isolated from S. alfredii in transgenic tobacco and Arabidopsis thaliana (A. thaliana)

73 conferred greater tolerance levels to Cd stress (Liu et al. 2016; Zhang et al. 2014; Zhang et al. 2016).

74 Overexpression of SaMT2 can chelate free cadmium in the cytoplasm and reduce the concentration of Cd.

75 SaCAX2 can transport Cd into vesicles and store. However, lipids that play important roles in abiotic stress

76 remain largely unknown in this species. Signaling lipids can accumulate proteins on the membrane in an

77 instant and affect the conformation and activity of proteins and metabolites in the cells, so that the plants

78 can grow normally under abiotic stress conditions (Hou et al. 2016). Many lipase and esterase sequences

79 have a pentapeptide GxSxG motif in which serine (S) is the central position of the conserved sequence.

80 However, the hydrolysis/lipolytic enzyme subfamily GDSL has conserved motifs with different structures,

81 the conserved amino acids are glycine (G), aspartic acid (D), $S$ and leucine $(L)$, and the active site serine is

82 located near the N-terminus. The GDSL esterase/lipase protein (GELP) family has been identified in

83 various plant species and is an attractive focus for scientists owing to their multifunctional nature in a wide

Peer] reviewing PDF | (2019:01:34381:1:1:NEW 6 Mar 2019) 
range of organisms. Currently, there are 104 known GELP family members in Arabidopsis (Lai et al. 2017; Ling 2008), and 130, 126, 96 and 57 family members in sorghum, Populus tomentosa, grape and Plutella xylostella, respectively (Volokita et al. 2011). GELPs are multifunctional hydrolytic enzymes that possess broad substrate specific and regiospecific activities. Consequently, the GELP enzymes are grouped in family II of the lipolytic enzymes (Akoh et al. 2004; Shakiba et al. 2016). They have four strictly conserved domains, I, II, III and V, which contain conserved Ser, Gly, Asn and His residues, respectively (Akoh et al. 2004; Molgaard et al. 2000). GELP family members have many functions in abiotic stress (Hong et al. 2008; Lee et al. 2009), morphogenesis (Ling et al. 2006), lipid metabolism (Brick et al. 1995) and seed development (Clauss et al. 2008; Riemann et al. 2007). AtGELP28 (SFAR2) and AtGELP59 (SFAR3) play key roles in plants under glucose-stress conditions (Chen et al. 2012). AtGELP60 (AtLTL1) enhances yeast tolerance to $\mathrm{LiCl}$ and might be involved in defense responses against pathogens (Naranjo et al. 2006). AtGLIP2 affects plant defense functions by inhibiting auxin responses (Lee et al. 2009).

However, S. alfredii GELP (SaGELP) gene has not yet been systematically identified under Cd stress or without Cd stress. Fortunately, the genome sequencing of $S$. alfredii has been completed by our group (unpublished), which enables the characterisation of the GELP family and their responses to Cd stress. In this study, we discovered 80 SaGELPs. A genome-wide bioinformatics analysis of the GELP family involved gene structures, phylogenetics and motif classification. In addition, the spatial-temporal expression patterns of SaGELP genes were determined under Cd-stress conditions. Finally, the heterologous expression of Sa13F.102 (SaGLIP8) in yeast increased Cd resistance and accumulation.

These results provide the foundation for further studies on the functions of the GELP family, and the regulatory mechanisms of SaGELPS under heavy metal-stress conditions. 
Materials and methods

106

107

108

110

111

112

113

114

115

116

117

118

119

120

121

122

123

\section{Plant materials and stress treatments}

Seedlings of the hyperaccumulator ecotype of $\mathrm{S}$. alfredii were collected from an old $\mathrm{Pb} / \mathrm{Zn}$ mining area in Quzhou City, Zhejiang Province, China. They were grown hydroponically in a growth chamber with day/night temperatures of $25^{\circ} \mathrm{C}$ and a $16-\mathrm{h} / 8$-h light/dark photoperiod. The seedlings were cultured in $1 / 2-$ strength Hoagland's solution for 4 weeks. Subsequently, the roots of the experimental seedlings were immersed in $100 \mu \mathrm{M} \mathrm{CdCl}_{2}$ as the stress treatment, while the seedlings of the control group were further cultured in 1/2-strength Hoagland's solution. Roots, stems and leaves were sampled at $0 \mathrm{~h}, 6 \mathrm{~h}$ and $7 \mathrm{~d}$. Three biological repeats per sample were taken at each time point and stored in a $-80^{\circ} \mathrm{C}$ refrigerator for subsequent use.

\section{Identification of SaGELPs in S. alfredii Hance}

The OrthoMCL algorithm ( $\mathrm{Li}$ et al. 2003) was used to analyze 16 species, S. alfredii and 15 other related species, Phalaenopsis equestris, Oryza sativa, A. thaliana, Populus trichocarpa, Amborella trichopoda, Rhodiola crenulata, Kalanchoe fedtschenkoi, Medicago truncatula, Brassica rapa, Solanum lycopersicum, Daucus carota, Coffea canephora, Nelumbo nucifera, Macleaya cordata and Ananas comosus. The HMMER search (https://www.ebi.ac.uk/Tools/hmmer/) (Finn et al. 2011) was conducted to identify and screen for possible SaGELPs containing Lipase_GDSL (Pfam: PF00657) domains in S. alfredii (Chen et al. 2018). The GELP homologous sequences in $A$. thaliana were obtained from TAIR (https://www.arabidopsis.org/index.jsp). All of the candidate SaGELPs were further confirmed by SMART 
124 (http://smart.embl-heidelberg.de/) (Letunic \& Bork 2018) according to AtGELPs. The basic information for

125 SaGELPs were predicted using ExPASy (https://web.expasy.org/protparam/) (Mariethoz et al. 2018),

126 including molecular weights, amino acid numbers and isoelectric point values.

127 Multiple sequence alignments and phylogenetic analyses

128 All of the validated SaGELP and selected AtGELP protein sequences were aligned with ClustalX in

129 MEGA5 using GONNET as the protein weight matrix, with a gap opening penalty of 10 and gap extension

130 penalty of 0.1 . Phylogenetic trees were constructed using the Neighbor-joining method with the following

131 parameters: text of phylogeny = bootstrap method; number of bootstrap replications = 1,000 ; and

132 gaps/missing data treatment = complete deletion. iTOL (http://itol.embl.de/upload.cgi) tools were used to

133 modify the phylogenetic trees (Letunic \& Bork 2016).

\section{Gene structure and conserved motif predictions}

135

136

137

138

139

140

141

142

Gene structure diagrams of SaGELPs were obtained from Gene Structure Display Server 2.0 (http://gsds.cbi.pku.edu.cn/) (Hu et al. 2014). Conserved motifs in SaGELPs sequences were identified using Multiple Expectation Maximization for Motif Elicitation (MEME, e value $<1 \mathrm{e}^{-10}$ ) services (http://meme-suite.org/tools/meme) (Bailey et al. 2015), with the following parameters: motif discovery = classic mode; number of repetitions $=0$ or 1 occurrence per sequence; maximum number of motifs $=50$; and optimum motif width $=6-100$ residues. The consensus blocks in conserved domains were constructed using WebLogo (http://weblogo.berkeley.edu/logo.cgi) (Crooks et al. 2004).

\section{Analysis of cis-regulatory elements from promoters}


143 The cis-regulatory elements in the promoters were predicted in the $1.5 \mathrm{~kb}$ upstream regions of all SaGELP

144 genes using the online website PlantCARE (http://bioinformatics.psb.ugent.be/webtools/plantcare/html/)

145 (Lescot et al. 2002). Different cis-responsive elements in the promoters were presented using RAST

146 (http://rsat.eead.csic.es/plants/feature-map_form.cgi).

\section{Co-expression network construction}

A weighted gene co-expression network analysis (WGCNA) was used to construct co-expression regulatory networks based on profiles of differentially expressed gene responses to Cd stress, as described by Han et al (Han et al. 2016). The Pearson's correlation coefficient of the Fragments Per Kilobase of transcript per Million fragments mapped (FPKM) value of each gene pair was calculated using the R programming language, with the correlation coefficient threshold set to 0.30 (Han et al. 2016). We screened the members of the SaGELP family and identified hub genes in the co-expression network (Langfelder et al. 2013). All eligible edges were classified according to their annotations, and we further analyze their associations with hub genes (Lotia et al. 2013). Finally, the co-expression subnetwork was visualized with Cytoscape v3.6.1 (Shannon et al. 2003).

\section{Total RNA isolation and expression analysis}

Total RNA of S. alfredii treated with $100 \mu \mathrm{M} \mathrm{CdCl}_{2}$ was extracted from all roots, stems and leaves, using an RNA extraction kit (NORGEN, Thorold, ON, Canada). RNase-free DNasel (New England BioLabs, Ipswich, MA, USA) was used to process genomic DNA and digest all samples. PrimeScript ${ }^{\mathrm{TM}}$ RT Master Mix (TaKaRa, Dalian, China) (Stephens et al. 2010) was used to produce the first-strand cDNA, which was 

stored at $-80^{\circ} \mathrm{C}$ for later use. $\operatorname{Taq}^{\text {TM }}$ (TaKaRa) reagent on the thermal circulator of an Applied Biosystems 7300 Real-Time PCR System

165 (Applied Biosystems, Foster City, CA, USA) (Chen et al. 2018). Sequences of primers used in qRT-PCR

166 are shown in Table S1. The relative expression level of each SaGELP gene was calculated based on the

167 comparison threshold period $\left(2^{-\triangle \triangle C T}\right)$ method, using SaUBC9 as an endogenous reference gene (Sang et 168 al. 2013). The heat map of the relative expression levels was constructed using online software at 169 OmicShare (http://www.omicshare.com/). The qRT-PCR products of the expected size were analyzed by $170 \quad 1.5 \%$ agarose gel electrophoresis.

\section{Heterologous expression of SaGLIP8 in yeast}

172 The specific primers SaGLIP8-F/R were used to amplify the open reading frame of SaGLIP8 (Table S1).

173 The purified PCR product was first inserted into the entry vector pDONR222 (Invitrogen, Carlsbad, CA, 174 USA), and then yeast expression vector pYES-DEST52-SaGLIP8 were constructed by gateway LR 175 reaction. The empty vector pYES2.0 was used as a control. Two vectors, expression vector pYES-

176 DEST52-SaGLIP8 as well as empty vector pYES2.0, were transformed into the Cd sensitive mutant strain

177 Saccharomyces cerevisiae ( $\Delta y c f 1)$ using the lithium acetate method (Liu et al. 2016). Positive colony

178 selection was performed in the solid medium with $50 \mu \mathrm{g} \cdot \mathrm{ml}^{-1}$ ampicillin and PCR reaction. The selected 179 positive clones in the yeast liquid were cultured to an OD600 value of $0.8-1.0$, and then spotted on SG-U 180 (synthetic galactose-uracil) solid medium containing concentrations of 0,15 and $30 \mu \mathrm{M} \mathrm{CdCl}$. The strains 
181 in SG-U liquid medium were diluted using the concent gradient $O_{600}=10^{0}, 10^{-1}, 10^{-2}, 10^{-3}, 10^{-4}$ and $10^{-5}$,

182 and incubated in a $28^{\circ} \mathrm{C}$ incubator for $3 \mathrm{~d}$ (Chen et al. 2017; Liu et al. 2016). In addition, two transformed

183 yeast cell strains were cultured on liquid SG-U medium containing $30 \mu \mathrm{M} \mathrm{CdCl}_{2}$ for $96 \mathrm{~h}$ at $28^{\circ} \mathrm{C}$ to

184 determine the $\mathrm{Cd}$ accumulation levels by the Inductively Coupled Plasma Mass Spectrometry (ICP-MS,

185 Nexlon 300D, Perkin Elmer, Shelton, CT, USA).

186

187

\section{Results}

\section{Eighty SaGELP family members were identified and classified into three clades}

A total of 80 SaGELPs and 56 pseudoenzymes (incomplete domain structure) were dug out (Table S2). All of the characteristics of the 80 SaGELP candidate genes are listed in Table 1, including the amino acid lengths, molecular weights and theoretical isoelectric point values. The coding sequence (CDS) lengths ranged from $900 \mathrm{bp}(\mathrm{Sa28F.37)}$ to 1,920 bp (Sa9F.272), with an average length of 1,131 bp. In total, 104 candidate sequences were obtained from $A$. thaliana through preliminary research, and the HMM analysis confirmed 101 AtGELP sequences and 4 pseudoenzymes (Table S3). We further used AtGELPs and SaGELPs to build phylogenetic tree. The SaGELP gene family is divided into clades I, II and III, and the numbers of subclades are 13,6 and 2, respectively (Fig. 1), consistent with a previous study of terrestrial plant AtGELPs (Volokita et al. 2011), showing that three branches, two major and one minor, existed in its phylogenetic tree.

\section{Gene structure and conserved motif analysis}

The genomic sequences ranged from $1,226 \mathrm{bp}$ (Sa24F.223) to 3,312 bp (Sa79F.143) (Table S4). The two 
200 genes (Sa116F.65 and Sa99F.11) had maximum number of exons (seven), while the three genes

201 (Sa39F.196, Sa9F.272 and Sa9F.81) contained only two exons. The gene structure analysis showed that

202 the average number of exons was five, within 56 SaGELP genes (70\%) containing five exons and four

203 introns (Fig. 2B, Table S5).

204 Conserved sequences and motifs represent important sites for enzymatic functions. Among the 23

205 discovered motifs (Fig. 2C, Table S6), we analyzed four conservative motifs in the SaGELPs, blocks I, II,

206 III and V (2, 5, 7 and 1, respectively), and the different blocks contained different motifs (Fig. 3, Table S7).

207 A total of 13 well-conserved motifs were found $\left(E\right.$ values $\left.<1 e^{-100}\right)$ in most SaGELP genes, while other

208 motifs were specific to individual SaGELPs (Table S7). Motifs 14 and 18 were only found in clade I, while

209 motifs 16 and 21 were only discovered in clade II. Motif 23 was unique in clades I and II, and motifs 20 and

21022 were distributed in clades I and III. The others motifs existed in all clades (Fig. 1A).

211 Analysis of cis-regulatory elements from promoters

212 The $1.5 \mathrm{~kb}$ upstream regulatory regions of the SaGELP genes were explored for stress-related regulatory

213 elements (Table S8). The cis-acting element analysis of all genes is shown in Fig. 4. We identified cis-

214 regulatory elements related to hormones, such as auxin, gibberellin, methyl jasmonate and ethylene. TC-

215 rich (ATTTTCTCCA) repeats are related to cis-acting elements involving in defense and stress response.

216 Meanwhile, some elements are also related to abiotic stress, such as heat shock elements (heat stress)

217 and MYB-binding (AACCTAA, MRE) sites (drought-inducibility).

\section{Co-expression network of SaGELPs}

219 A large number of hub genes regulate potential target genes, including those related to general tolerance 
220

221

222

223

224

225

226

227

228

229

230

231

232

233

234

235

236

237

238

239

mechanisms and responses to Cd stress. Here, a total of 13 hub genes related to SaGELPS were obtained,

as well as potential edge genes. The co-expression regulatory network involved 13 hub genes and 5 regulated different functional groups from GO (Gene Ontology) annotation (Table S7). Most of the coexpressed genes are involved in metabolic processes, growth and development, catalytic activity and biological regulation, indicating that SaGELPs have multiple functions in plants. We selected the edge genes involved in Cd tolerance from several regulatory networks, including cell wall and defense function, lipid and esterase, stress and tolerance, transport and transcription factor activity (Table S7).

As shown in Fig. 5, the major categories were transport (254 edges), transcription factor (112 edges), lipid and esterase (63 edges), cell wall and defense function (24 edges), and stress and tolerance (6 edges).

The hub gene SaOF.898 had the largest module in the Cd response gene co-expression network, with 128 nodes, including $60,29,19,7$ and 3 nodes related to transport, transcription factor, lipid and esterase, cell wall and defense function, and stress and tolerance, respectively. Other hub genes were also associated with different biological functions. For example, Sa13F.102 was mainly related to lipid, esterase, cell wall and defense function, while Sa26F.146 was mainly involved transport function. Therefore, SaGELPs might be involved in the induction of stress signals and function by activating transcription factors to regulate genes involved in metal transport. In addition, they was related to the enhancement of the plant's resistance to heavy metals.

\section{Tissue expression patterns and Cd response profiles}

We used qRT-PCR to understand the functions of the SaGELP genes in $S$. alfredii and the tissue expression pattern under Cd-stress conditions at three time points $(0 \mathrm{~h}, 6 \mathrm{~h}$ and $7 \mathrm{~d})$. The tissues 
240

241

242

expression profiles of the genes were converted into a heat map on the basis of their expression levels

(Fig. 6). All of the expression levels of SaGELP genes could be divided into the following three cases: 1)

75 genes expression significantly up-regulated at $6 \mathrm{~h}$ and decreased at $7 \mathrm{~d}$ in root (such as Sa28F.36,

Sa5F.25 and Sa46F.20), stem (such as Sa27F.42, Sa45F.55 and Sa314F.6) and leaf (such as Sa13F.118,

SaOF.41 and Sa42F.134); 2) 7 up-regulated expression in stems and leaves (such as Sa10F.217); and 3)

12 down-regulated trends in roots, stems and leaves (such as Sa105F.31, Sa7F.458 and Sa184F.22).

Furthermore, the gene expression levels were greatly different in untreated samples (without Cd treatment). We performed a data analysis on the hub genes in roots, stems and leaves. Sa13F.102, Sa28F.36 and Sa5F.25 were constitutively expressed at relatively high levels in root (Fig. 7A), while the three most highly expressed SaGELPs in the stem were Sa13F.102, Sa5F.25 and Sa29F.188.1 (Fig. 7B), and the three most highly expressed SaGELPs in the leaf were Sa13F.102, Sa95F.131 and Sa29F.188.1

(Fig. 7C). Sa13F.102 had the highest expression level in all tissues. Meanwhile, the results from qRT-PCR gel image were in accordance with gene expression levels (Fig. S2).

\section{SaGLIP8 heterologous expression enhanced Cd tolerance and accumulation in}

\section{yeast}

Due to the recent relationship between the Sa13F.102 gene and At5G45670.1 (AtGLIP8) from the above phylogenetic tree (Fig. S1), we designated Sa13F.102 gene as SaGLIP8. As a hub gene in the coexpression network, Sa13F.102 (SaGLIP8) was selected for functional verification for its strong induction in response to Cd stress, which implied vital roles in the Cd response in all three tissues. SaGLIP8 gene was expressed in the Cd sensitive mutant strain Saccharomyces cerevisiae ( $\triangle y c f 1)$. The SaGLIP8- 
260

261

262

263

264

265

266

267

268

269

270

271

272

273

274

275

276

277

278

279

overexpressive yeast grew better than the pYES2.0 yeast on a medium containing 15 and $30 \mu \mathrm{M} \mathrm{CdCl}$,

suggesting that the SaGLIP8 gene could increase Cd tolerance in yeast (Fig. 8A). Cd concentration

measurements, with pYES2.0 as the control, revealed that the Cd content of SaGLIP8-overexpressive

yeast was significantly greater than that of pYES2.0, and the difference between the two was extremely

significant $(P=0.01)$ (Fig. 8B).

\section{Discussion}

Plants can accumlimate to environmental pollution such as heavy metals through activating the expression

of corresponding proteins involving in stress response, such as phytochelatins (PCs) and metallothioneins

(MTs) (Hasan et al. 2017). Hyperaccumulator plants accumulate heavy metals in the body and exhibit

enhanced tolerance levels; consequently, they can be used for phytoremediation and other purposes

(DalCorso et al. 2013; Tian et al. 2016). S. alfredii is a hyper-accumulation plant, which absorbs and accumulates $\mathrm{Cd}$ from the soil. Thus, it is a promising candidate plant species to alleviate and solve soil

pollution problems (Clemens et al. 2013). There have been many reports on Cd absorption and dynamic

balance in S. alfredii (Liu et al. 2016; Tian et al. 2017); however, the molecular mechanism underlying Cd

detoxification in S. alfredii remains poorly understood.

GELP family members have been reported in many plant species, and have many roles, including in abiotic stress responses and defense functions (Abdelkafi et al. 2009; Cao et al. 2018; Dong et al. 2016;

Lai et al. 2017; Tan et al. 2014). GLIP1 in pepper can participate in wound defense responses (Hong et al. 2008). OsGLIP1 and OsGLIP2 proteins from O. sativa are located in lipid droplets and endoplasmic reticulum membranes and play a key role in lipid metabolism and immune response (Gao \& Yin 2017). The 
280

281

282

GELP family plays important roles in plant abiotic stress responses, but research on their roles under Cdstress conditions was limited. In this study, 80 SaGELP genes of $S$. alfredii were identified. A phylogenetic analysis showed that SaGELPs could be divided into three main groups (Fig. 1), which was consistent with the classifications reported by previous researchers (Lai et al. 2017; Ling et al. 2006). The responses of the GDSL family to biotic and abiotic stresses had been studied (Hong et al. 2008; Shakiba et al. 2016), but there were few studies on the effects of heavy metal contamination. Therefore, it was necessary to investigate the effects of heavy metals on the SaGELP family of genes.

In Brassica napus L., the BnLIP2 gene was expressed in a tissue-specific manner and was abundantly expressed during seed germination (Ling et al. 2006). Was the GELP family also expressed in a tissuespecific manner in S. alfredii ? In this experiment, we treated roots of $S$. alfredii seedlings with $100 \mu \mathrm{M}$ $\mathrm{CdCl}_{2}$ and used three different time points to construct a heat map to observe expression changes. The roots, stems and leaves under $0 \mathrm{~h}$ were used the $\mathrm{qRT}$ - PCR. Then analyze the results, used the online software to draw the heat map. Relative expression values were calculated by Z-score normalization. Most of the genes showed significant changes, which were related to $S$. alfredii's ability to co-excessively accumulate Cd (Fig. 6). The hub gene Sa13F.102 was abundantly expressed in all tissues (roots, stems and leaves) under normal conditions $(0 \mathrm{~h})$, and other hub genes were abundantly expressed in specific tissues, such as Sa28F.36's expression in roots and Sa5F.25's expression in stems (Fig. 7). Thus, some SaGELP gene family members were expressed in a tissue-specific manner. After $\mathrm{Cd}$ treatments, some genes also showed specificity of expression, such as Sa28F.36 and Sa28F.38, which were only upregulated in roots, Sa10F.217 and Sa29F.343, which were only up-regulated in stems, and SaOF.41 and Sa12F.49, which were only up-regulated in leaves. As members of an esterase or lipase gene family 
301 (Akoh et al. 2004), some SaGELP genes might be associated with cell wall synthesis (Zhang et al. 2017)

302 and stress (Shakiba et al. 2016). Consequently, we selelcted 13 hub genes that had edge genes with

303 these or related functions according to their gene ontology classification and constructed a co-expression

304 regulatory network (Fig. 5). Most of the identified genes were involved in transport (254 edges) and a few

305 were associated with stress responses (6 edges).

306 We predicted that SaGLIP8 encodes an extracellular protein. As shown in Fig. 8, SaGLIP8 could increase

307 the Cd tolerance and content in transgenic yeast. Thus, we hypothesize that this protein may function like

308 the OsGLIP1 and OsGLIP2 proteins. Some reported GEIP genes can be regulated in a variety of ways to

309 enhance their defense functions. Brittle leaf sheath1 (BS1) in rice is a member of the GELP family and is

310 involved in the formation of this cell wall and plays an important role in the defense function of plants

311 (Zhang et al. 2017). The GELP genes especially SaGLIP8 in S. alfredii can also be regulated by several

312 means, which may improve Cd tolerance.

\section{Conclusions}

Based on genomic data of $S$. alfredii, we conducted phylogenetic analyses, as well as conserved domain, motif and expression profiling of the GELP family under Cd-stress conditions. The phylogenetic trees were constructed by combining the $A$. thaliana and $S$. alfredii GELP family genes, which indicated that the associated domains were conserved during evolution. According to the structural and phylogenetic characteristics of the SaGELP sequences, they were divided into three clades. Most of the genes were responsive to $\mathrm{Cd}$ stress. In total, 13 hub genes were obtained, and a co-expression regulatory subnetwork 
321

322

cloned into an expression vector and transformed into yeast. SaGLIP8 enhanced Cd tolerance and accumulation in yeast. This result indicated the roles of GELPs in plant responses to heavy metal exposure and provides a theoretical basis for further studies of the SaGELP family's functions.

\section{References}

Abdelkafi S, Ogata H, Barouh N, Fouquet B, Lebrun R, Pina M, Scheirlinckx F, Villeneuve P, and Carriere F. 2009. Identification and biochemical characterization of a GDSL-motif carboxylester hydrolase from Carica papaya latex. Biochim Biophys Acta 1791:1048-1056.

Akoh CC, Lee GC, Liaw YC, Huang TH, and Shaw JF. 2004. GDSL family of serine esterases/lipases. Prog Lipid Res 43:534-552.

Bailey TL, Johnson J, Grant CE, and Noble WS. 2015. The MEME Suite. Nucleic Acids Res 43:W39-49.

Brick DJ, Brumlik MJ, Buckley JT, Cao JX, Davies PC, Misra S, Tranbarger TJ, and Upton C. 1995. A new family of lipolytic plant enzymes with members in rice, arabidopsis and maize. FEBS Lett 377:475-480.

Cao Y, Han Y, Meng D, Abdullah M, Yu J, Li D, Jin Q, Lin Y, and Cai Y. 2018. Expansion and evolutionary patterns of GDSL-type esterases/lipases in Rosaceae genomes. Funct Integr Genomics 18:673-684.

Chen M, Du X, Zhu Y, Wang Z, Hua S, Li Z, Guo W, Zhang G, Peng J, and Jiang L. 2012. Seed Fatty Acid Reducer acts downstream of gibberellin signalling pathway to lower seed fatty acid storage in Arabidopsis. Plant Cell Environ 35:2155-2169.

Chen S, Han X, Fang J, Lu Z, Qiu W, Liu M, Sang J, Jiang J, and Zhuo R. 2017. Sedum alfredii SaNramp6 Metal Transporter Contributes to Cadmium Accumulation in Transgenic Arabidopsis thaliana. Scientific Reports 7:13318.

Chen SS, Jiang J, Han XJ, Zhang YX, and Zhuo RY. 2018. Identification, Expression Analysis of the Hsf Family, and Characterization of Class A4 in Sedum Alfredii Hance under Cadmium Stress. international journal of molecular sciences 19.

Clauss K, Baumert A, Nimtz M, Milkowski C, and Strack D. 2008. Role of a GDSL lipase-like protein as sinapine esterase in Brassicaceae. Plant J 53:802-813.

Clemens S, Aarts MG, Thomine S, and Verbruggen N. 2013. Plant science: the key to preventing slow cadmium poisoning. Trends Plant Sci 18:92-99.

DalCorso G, Manara A, and Furini A. 2013. An overview of heavy metal challenge in plants: from roots to shoots. Metallomics 5:1117-1132.

Dong X, Yi H, Han CT, Nou IS, and Hur Y. 2016. GDSL esterase/lipase genes in Brassica rapa L.: genome-wide identification and expression analysis. Mol Genet Genomics 291:531-542.

Finn RD, Clements J, and Eddy SR. 2011. HMMER web server: interactive sequence similarity searching. Nucleic Acids Res 39:W29-37.

Gao J, Sun L, Yang X, and Liu JX. 2014. Transcriptomic analysis of cadmium stress response in the heavy metal hyperaccumulator Sedum alfredii Hance. PLoS One 8:e64643.

Gao M, and Yin X. 2017. GDSL lipases modulate immunity through lipid homeostasis in rice. PLoS Pathogens 
13:e1006724.

Han X, Yin H, Song X, Zhang Y, Liu M, Sang J, Jiang J, Li J, and Zhuo R. 2016. Integration of small RNAs, degradome and transcriptome sequencing in hyperaccumulator Sedum alfredii uncovers a complex regulatory network and provides insights into cadmium phytoremediation. Plant Biotechnol J 14:1470-1483.

Hasan MK, Cheng Y, Kanwar MK, Chu XY, Ahammed GJ, and Qi ZY. 2017. Responses of Plant Proteins to Heavy Metal Stress-A Review. Front Plant Sci 8:1492.

Hong JK, Choi HW, Hwang IS, Kim DS, Kim NH, Choi DS, Kim YJ, and Hwang BK. 2008. Function of a novel GDSL-type pepper lipase gene, CaGLIP1, in disease susceptibility and abiotic stress tolerance. Planta 227:539-558.

Hou Q, Ufer G, and Bartels D. 2016. Lipid signalling in plant responses to abiotic stress. Plant Cell Environ 39:10291048.

Hu B, Jin J, Guo AY, Zhang H, Luo J, and Gao G. 2014. GSDS 2.0: an upgraded gene feature visualization server. Bioinformatics 31:1296.

Jarup L, and Akesson A. 2009. Current status of cadmium as an environmental health problem. Toxicol Appl Pharmacol 238:201-208.

Lai CP, Huang LM, Chen LO, Chan MT, and Shaw JF. 2017. Genome-wide analysis of GDSL-type esterases/lipases in Arabidopsis. Plant Mol Biol 95:181-197.

Langfelder P, Mischel PS, and Horvath S. 2013. When is hub gene selection better than standard meta-analysis? PLoS One 8:e61505.

Lee DS, Kim BK, Kwon SJ, Jin HC, and Park OK. 2009. Arabidopsis GDSL lipase 2 plays a role in pathogen defense via negative regulation of auxin signaling. Biochem Biophys Res Commun 379:1038-1042.

Lescot M, Dehais P, Thijs G, Marchal K, Moreau Y, Van de Peer Y, Rouze P, and Rombauts S. 2002. PlantCARE, a database of plant cis-acting regulatory elements and a portal to tools for in silico analysis of promoter sequences. Nucleic Acids Res 30:325-327.

Letunic I, and Bork P. 2016. Interactive tree of life (iTOL) V3: an online tool for the display and annotation of phylogenetic and other trees. Nucleic Acids Res 44:W242-245.

Letunic I, and Bork P. 2018. 20 years of the SMART protein domain annotation resource. Nucleic Acids Res 46:D493-d496.

Li L, Stoeckert CJ, Jr., and Roos DS. 2003. OrthoMCL: identification of ortholog groups for eukaryotic genomes. Genome Res 13:2178-2189.

Li Z, Han X, Song X, Zhang Y, Jiang J, Han Q, Liu M, Qiao G, and Zhuo R. 2017. Overexpressing the Sedum alfredii $\mathrm{Cu} / \mathrm{Zn}$ Superoxide Dismutase Increased Resistance to Oxidative Stress in Transgenic Arabidopsis. Front Plant Sci 8:1010.

Ling H. 2008. Sequence analysis of GDSL lipase gene family in Arabidopsis thaliana. Pak J Biol Sci 11:763-767.

Ling H, Zhao J, Zuo K, Qiu C, Yao H, Qin J, Sun X, and Tang K. 2006. Isolation and expression analysis of a GDSL-like lipase gene from Brassica napus L. J Biochem Mol Biol 39:297-303.

Liu H, Zhao H, Wu L, Liu A, Zhao FJ, and Xu W. 2017. Heavy metal ATPase 3 (HMA3) confers cadmium hypertolerance on the cadmium/zinc hyperaccumulator Sedum plumbizincicola. New Phytol 215:687-698.

Liu M, Qiu W, He X, Zheng L, Song X, Han X, Jiang J, Qiao G, Sang J, Liu M, and Zhuo R. 2016. Functional Characterization of a Gene in Sedum alfredii Hance Resembling Rubber Elongation Factor Endowed with Functions Associated with Cadmium Tolerance. Front Plant Sci 7:965.

Liu Y, Li Y, Liu K, and Shen J. 2014. Exposing to cadmium stress cause profound toxic effect on microbiota of the 
mice intestinal tract. PLoS One 9:e85323.

Lotia S, Montojo J, Dong Y, Bader GD, and Pico AR. 2013. Cytoscape app store. Bioinformatics 29:1350-1351.

Mariethoz J, Alocci D, Gastaldello A, Horlacher O, Gasteiger E, Rojas-Macias M, and Karlsson NG. 2018. Glycomics@ExPASy: Bridging the gap. Mol Cell Proteomics 17:2164-2176.

Messner B, and Bernhard D. 2010. Cadmium and cardiovascular diseases: cell biology, pathophysiology, and epidemiological relevance. Biometals 23:811-822.

Molgaard A, Kauppinen S, and Larsen S. 2000. Rhamnogalacturonan acetylesterase elucidates the structure and function of a new family of hydrolases. Structure 8:373-383.

Nair AR, Degheselle O, Smeets K, Van Kerkhove E, and Cuypers A. 2013. Cadmium-Induced Pathologies: Where Is the Oxidative Balance Lost (or Not)? Int J Mol Sci 14:6116-6143.

Naranjo MA, Forment J, Roldan M, Serrano R, and Vicente O. 2006. Overexpression of Arabidopsis thaliana LTL1, a salt-induced gene encoding a GDSL-motif lipase, increases salt tolerance in yeast and transgenic plants. Plant Cell Environ 29:1890-1900.

Oono Y, Yazawa T, Kawahara Y, Kanamori H, Kobayashi F, Sasaki H, Mori S, Wu J, Handa H, Itoh T, Matsumoto T. 2014. Genome-wide transcriptome analysis reveals that cadmium stress signaling controls the expression of genes in drought stress signal pathways in rice. PLoS One 9:e96946.

Pilon-Smits E. 2005. Phytoremediation. Annu Rev Plant Biol 56:15-39.

Rascio N, and Navari-Izzo F. 2011. Heavy metal hyperaccumulating plants: how and why do they do it? And what makes them so interesting? Plant Sci 180:169-181.

Riemann M, Gutjahr C, Korte A, Riemann M, Danger B, Muramatsu T, Bayer U, Waller F, Furuya M, and Nick P. 2007. GER1, a GDSL motif-encoding gene from rice is a novel early light- and jasmonate-induced gene. Plant Biol (Stuttg) 9:32-40.

Sang J, Han X, Liu M, Qiao G, Jiang J, and Zhuo R. 2013. Selection and validation of reference genes for real-time quantitative PCR in hyperaccumulating ecotype of Sedum alfredii under different heavy metals stresses. PLoS One 8:e82927.

Satarug S, Garrett SH, Sens MA, and Sens DA. 2011. Cadmium, environmental exposure, and health outcomes. Cien Saude Colet 16:2587-2602.

Shakiba MH, Ali MS, Rahman RN, Salleh AB, and Leow TC. 2016. Cloning, expression and characterization of a novel coldadapted GDSL family esterase from Photobacterium sp. strain J15. Extremophiles 20:44-55.

Shannon P, Markiel A, Ozier O, Baliga NS, Wang JT, Ramage D, Amin N, Schwikowski B, and Ideker T. 2003. Cytoscape: a software environment for integrated models of biomolecular interaction networks. Genome Res 13:2498-2504.

Sharma SS, Dietz KJ, and Mimura T. 2016. Vacuolar compartmentalization as indispensable component of heavy metal detoxification in plants. Plant Cell Environ 39:1112-1126.

Stephens KW, Hutchins RJ, and Dauphin LA. 2010. Cross-platform evaluation of commercial real-time reverse transcription PCR master mix kits using a quantitative 5 'nuclease assay for Ebola virus. Mol Cell Probes 24:370-375.

Tan X, Yan S, Tan R, Zhang Z, Wang Z, and Chen J. 2014. Characterization and expression of a GDSL-like lipase gene from Brassica napus in Nicotiana benthamiana. Protein J 33:18-23.

Tian S, Xie R, Wang H, Hu Y, Ge J, Liao X, Gao X, Brown P, Lin X, and Lu L. 2016. Calcium Deficiency Triggers Phloem Remobilization of Cadmium in a Hyperaccumulating Species. Plant Physiol 172:2300-2313. 
439

440

441

442

443

444

445

446

447

448

449

450

451

452

453

454

455

456

457

458

459

460

461

462

463

464

465
Tian S, Xie R, Wang H, Hu Y, Hou D, Liao X, Brown PH, Yang H, Lin X, Labavitch JM, Lu L. 2017. Uptake, sequestration and tolerance of cadmium at cellular levels in the hyperaccumulator plant species Sedum alfredii. $J$ Exp Bot 68:2387-2398.

Volokita M, Rosilio-Brami T, Rivkin N, and Zik M. 2011. Combining comparative sequence and genomic data to ascertain phylogenetic relationships and explore the evolution of the large GDSL-lipase family in land plants. Mol Biol Evol 28:551-565.

Xing Y, Peng H, Gao L, Luo A, and Yang X. 2013. A compound containing substituted indole ligand from a hyperaccumulator Sedum alfredii Hance under Zn exposure. Int J Phytoremediation 15:952-964.

Zhang B, Zhang L, Li F, Zhang D, Liu X, Wang H, Xu Z, and Chu C. 2017. Control of secondary cell wall patterning involves xylan deacetylation by a GDSL esterase.Nature Plants 3:17017.

Zhang J, Zhang M, Tian S, Lu L, Shohag MJ, and Yang X. 2014. Metallothionein 2 (SaMT2) from Sedum alfredii Hance confers increased Cd tolerance and accumulation in yeast and tobacco. PLoS One 9:e102750.

Zhang M, Senoura T, Yang X, and Nishizawa NK. 2011. Functional analysis of metal tolerance proteins isolated from $\mathrm{Zn} / \mathrm{Cd}$ hyperaccumulating ecotype and non-hyperaccumulating ecotype of Sedum alfredii Hance. FEBS Lett 585:2604-2609.

Zhang M, Zhang J, Lu LL, Zhu ZQ, and Yang XE. 2016. Functional analysis of CAX2-like transporters isolated from two ecotypes of Sedum alfredii. Biologia Plantarum 60:37-47.

Zhu H, Ai H, Cao L, Sui R, Ye H, Du D, Sun J, Yao J, Chen K, and Chen L. 2018. Transcriptome analysis providing novel insights for Cd-resistant tall fescue responses to Cd stress. Ecotoxicol Environ Saf 160:349-356. 
467 Supporting Information:

468

469

470

471

472

473

474

475

476

477

478

479

480

481

482

483

484
Fig. S1 Phylogenetic relationship of S. alfredii Hance 13F.102 and the AtGELP gene family. The tree was generated with ClustalW and MEGA 5.0 software using the Neighbor-joining method. The number of bootstrap replications is 1,000 .

Fig. S2 The qRT-PCR gel image of $13 \mathrm{~S}$. alfredii GELP hub genes in different tissues under normal conditions. (A) root; (B) stem; (C) leaf. UBC9 is reference gene of S. alfredii. M stands for maker 2000.

Relative expression values were calculated by Z-score normalization. Green and red represent the low and high expression levels, respectively. The names of the samples are shown at the bottom.

Table S1 qRT-PCR primer sequences.

Table S2 Conding sequences and amino acid of all genes.

Table S3 The sequences of AtGELPs.

Table S4 Genomic sequences of SaGELPs.

Table S5 The number of exons and introns in SaGELPs.

Table S6 Conserved motifs identified in the SaGLIP proteins.

Table S7 The hub genes and edge genes.

Table S8 The promoter sequences of SaGELPs. 
485

486

487 Table legend:

488 Table 1 Analysis of amino acid sequence information of S. alfredii hance GELP family.

489 CDS: Coding sequence; AA: amino acid; pl: isoelectric point.

490

491

492

493

494

495

496

497

498

499 
Figure legends

Fig. 1 Phylogenetic relationships among the $S$. alfredii and Arabidopsis thaliana GELP families.

The tree was generated with ClustalW and MEGA 5.0 software using the Neighbor-joining method.

SaGELPs are labeled in red, and AtGELPs are labeled in black. Different clades are represented by different colored backgrounds. Different sub-branches are represented by different colored branching lines. The pale blue solid circle represents leaf sorting. The size of circle corresponds to the bootstrap value.

Fig. 2 Gene structures and motif compositions of the GELP family in S. alfredii. (A) The phylogenetic tree of SaGELP amino acid sequences was inferred using the Neighbor-joining method and 1000 bootstrap replicates; (B) Gene structures of SaGELPS. Yellow boxes represent CDS, blue boxes represent upstream or downstream, lines represent introns; (C) Schematic representation of each of the conserved motifs in the SaGELPs selected by the MEME online tool. Different motifs are represented by different colored boxes.

Fig. 3 Four conserved motifs of S. alfredii GELPs. The four consensus blocks I, II, III and V represent the conserved motifs in the amino acid sequences of the GDSL family, and are numbered based on their location from the $\mathrm{N}$ to $\mathrm{C}$ terminal. Triangles represent the conserved catalytic residues in conserved domains. Ser (S), Gly (G), Asn (N) and His (H) are conserved residues. 
519 Fig. 4 Cis-acting element analysis of promoters from the GELP family in S. alfredii. Different

520 elements are represented by different colored boxes. The box size corresponds to the element's sequence

521 length.

Fig. 5 Co-expression network of S. alfredii GELP genes. Nodes indicate genes, and edges indicate significant co-expression events between genes. Target genes involved in the same process are grouped together, and different groups are distinguished by different colors.

Fig. 6 Expression profiles of S. alfredii GELPs in root (R), stem (S) and leaf (L) under normal and

cadmium (Cd)-stress conditions. The heat map shows the expression of 80 SaGELP genes. Each small square represents a gene, and its color represents the expression of the gene. The greater the expression, the darker the color (red, up-regulated; green, down-regulated). The "0" represents the control without Cd stress. The stress time were six hours $(6 \mathrm{~h})$ and seven days $(7 \mathrm{~d})$.

Fig. 7 The expression profiles of $13 \mathrm{~S}$. alfredii GELP hub genes in different tissues under normal

conditions. (A) root; (B) stem; (C) leaf. The expression level of the control, Sa26F.146, (y-axis "Relative three independent biological replicates.

Fig. 8 Overexpression of S. alfredii Hance GLIP8 increases the cadmium (Cd) tolerance and

accumulation in yeast. (A) the growth of $\Delta y c f 1$ yeast mutants transformed with the empty vector

pYES2.0 or with pYES-DEST52 harboring SaGLIP8; (B) the accumulation of Cd in $\Delta y c f 1$ yeast cells. Bars 


\section{Table $\mathbf{1}$ (on next page)}

Analysis of amino acid sequence information of Sedum alfredii hance GELP family

CDS: Coding sequence; AA: amino acid; pl: isoelectric point. 


\begin{tabular}{|c|c|c|c|c|c|}
\hline Name & GeneBank accession no. & CDS Length (bp) & Number of AA & Molecular weight & Theoretical pI \\
\hline $\mathrm{Sa} 0 \mathrm{~F} .11$ & MK440731 & 1089 & 362 & 40634.89 & 8.38 \\
\hline $\mathrm{Sa0F} .223$ & MK440732 & 1143 & 380 & 41846.12 & 9.22 \\
\hline $\mathrm{Sa0F} .262$ & MK440733 & 1056 & 351 & 39364.84 & 6.45 \\
\hline $\mathrm{Sa} 0 \mathrm{~F} .41$ & MK440734 & 1089 & 362 & 40526.79 & 8.46 \\
\hline Sa0F.898 & MK440735 & 1113 & 370 & 42075.78 & 4.93 \\
\hline Sa105F.31 & MK440736 & 1095 & 364 & 40161.02 & 9.24 \\
\hline Sa10F.217 & MK440737 & 1104 & 367 & 40867.46 & 5.07 \\
\hline Sa10F.441 & MK440738 & 1056 & 351 & 39493.17 & 5.91 \\
\hline Sa110F.22 & MK440739 & 1197 & 398 & 44141.56 & 5.19 \\
\hline Sa116F.110 & MK440740 & 1155 & 384 & 42046.70 & 5.80 \\
\hline Sa116F.65 & MK440741 & 1320 & 439 & 49170.05 & 8.67 \\
\hline Sa121F.27 & MK440742 & 1086 & 361 & 39895.98 & 8.51 \\
\hline Sa129F.2 & MK440743 & 1092 & 363 & 39815.06 & 8.87 \\
\hline Sa12F.49 & MK440744 & 1092 & 363 & 38720.53 & 5.16 \\
\hline Sa136F.34 & MK440745 & 1134 & 377 & 41922.28 & 8.34 \\
\hline Sa13F.102 & MK440746 & 1098 & 365 & 40683.49 & 9.54 \\
\hline Sa13F.118 & MK440747 & 1125 & 374 & 41088.11 & 8.51 \\
\hline Sa14F.252.1 & MK440748 & 1188 & 395 & 44124.62 & 6.04 \\
\hline Sa184F.22 & MK440749 & 1089 & 362 & 40195.00 & 5.28 \\
\hline
\end{tabular}




\begin{tabular}{|c|c|c|c|c|c|}
\hline Sa18F.151 & MK440750 & 1164 & 387 & 42818.94 & 8.86 \\
\hline Sa1F.117 & MK440751 & 1032 & 343 & 37924.76 & 9.22 \\
\hline Sa207F.22 & MK440752 & 1119 & 372 & 41258.41 & 8.65 \\
\hline Sa20F.120 & MK440753 & 1359 & 452 & 50719.60 & 9.00 \\
\hline Sa217F.43 & MK440754 & 999 & 332 & 36974.89 & 5.43 \\
\hline Sa248F.34 & MK440755 & 1062 & 353 & 37905.99 & 6.64 \\
\hline Sa24F.223 & MK440756 & 1059 & 352 & 38951.99 & 5.69 \\
\hline Sa24F.262 & MK440757 & 1089 & 362 & 39028.77 & 8.59 \\
\hline Sa258F.41 & MK440758 & 1095 & 364 & 40739.54 & 5.32 \\
\hline Sa26F.146 & MK440759 & 1131 & 376 & 40913.88 & 7.52 \\
\hline Sa26F.158 & MK440760 & 1092 & 363 & 40043.83 & 5.06 \\
\hline Sa27F.42 & MK440761 & 1149 & 382 & 42465.01 & 6.25 \\
\hline Sa28F.36 & MK440762 & 1119 & 372 & 40698.35 & 5.37 \\
\hline Sa28F.37 & MK440763 & 900 & 299 & 32786.50 & 5.95 \\
\hline Sa28F.38 & MK440764 & 1098 & 365 & 40515.96 & 4.98 \\
\hline Sa28F.39 & MK440765 & 1095 & 364 & 39581.04 & 6.02 \\
\hline Sa29F.188.1 & MK440766 & 1146 & 381 & 40894.65 & 8.72 \\
\hline Sa29F.343 & MK440767 & 1068 & 355 & 40295.08 & 4.84 \\
\hline Sa2F.358 & MK440768 & 1053 & 350 & 38328.89 & 9.14 \\
\hline Sa314F.6 & MK440769 & 1113 & 370 & 40383.85 & 8.65 \\
\hline Sa32F.165 & MK440770 & 1092 & 363 & 39855.27 & 6.98 \\
\hline Sa33F.119 & MK440771 & 1179 & 392 & 43638.93 & 6.24 \\
\hline
\end{tabular}




\begin{tabular}{|c|c|c|c|c|c|}
\hline Sa36F.86 & MK440772 & 1116 & 371 & 40462.25 & 6.74 \\
\hline Sa39F.196 & MK440773 & 1149 & 382 & 42338.07 & 8.44 \\
\hline Sa39F.291 & MK440774 & 1206 & 401 & 44639.75 & 5.03 \\
\hline Sa3F.277 & MK440775 & 1104 & 367 & 39972.74 & 8.30 \\
\hline Sa3F.554 & MK440776 & 1107 & 368 & 41055.21 & 6.26 \\
\hline Sa42F.134 & MK440777 & 1107 & 368 & 40177.26 & 8.73 \\
\hline Sa45F.55 & MK440778 & 1074 & 357 & 39953.75 & 9.41 \\
\hline Sa46F.118 & MK440779 & 1146 & 381 & 43149.62 & 9.44 \\
\hline Sa46F.20 & MK440780 & 1095 & 364 & 40835.13 & 8.38 \\
\hline Sa46F.270 & MK440781 & 1131 & 376 & 41403.07 & 8.31 \\
\hline Sa46F.31 & MK440782 & 1089 & 362 & 40230.55 & 9.20 \\
\hline Sa47F.286 & MK440783 & 1140 & 379 & 42340.79 & 5.50 \\
\hline Sa55F.146 & MK440784 & 1167 & 388 & 42873.82 & 7.05 \\
\hline Sa56F.143 & MK440785 & 1104 & 367 & 40895.18 & 8.48 \\
\hline Sa57F.156 & MK440786 & 1137 & 378 & 41578.62 & 5.45 \\
\hline Sa5F.25 & MK440787 & 1056 & 351 & 38963.46 & 8.38 \\
\hline Sa5F.658 & MK440788 & 1089 & 362 & 40100.03 & 8.81 \\
\hline Sa64F.90 & MK440789 & 1107 & 368 & 40530.32 & 5.98 \\
\hline Sa66F.83 & MK440790 & 1188 & 395 & 43742.49 & 5.59 \\
\hline Sa6F.163.1 & MK440791 & 1197 & 398 & 44232.55 & 5.11 \\
\hline Sa6F.233 & MK440792 & 1089 & 362 & 39775.35 & 8.72 \\
\hline Sa72F.20 & MK440793 & 1068 & 355 & 38596.68 & 8.71 \\
\hline
\end{tabular}




\begin{tabular}{|c|c|c|c|c|c|}
\hline Sa79F.141 & MK440794 & 1164 & 387 & 42555.48 & 5.52 \\
\hline Sa79F.142 & MK440795 & 1122 & 373 & 40791.61 & 5.86 \\
\hline Sa79F.143 & MK440796 & 1131 & 376 & 41701.89 & 8.07 \\
\hline Sa7F.179 & MK440797 & 1071 & 356 & 39256.81 & 8.39 \\
\hline Sa7F.184 & MK440798 & 1107 & 368 & 40817.82 & 5.55 \\
\hline Sa7F.458 & MK440799 & 1104 & 367 & 40610.49 & 9.19 \\
\hline Sa7F.504 & MK440800 & 1143 & 380 & 42515.73 & 9.40 \\
\hline Sa81F.175 & MK440801 & 1107 & 368 & 40903.24 & 9.06 \\
\hline Sa82F.57 & MK440802 & 1074 & 357 & 38294.41 & 6.58 \\
\hline Sa84F.200 & MK440803 & 1140 & 379 & 41043.23 & 5.00 \\
\hline Sa86F.120 & MK440804 & 1098 & 365 & 40085.52 & 7.55 \\
\hline Sa92F.24 & MK440805 & 1128 & 375 & 42017.14 & 5.68 \\
\hline Sa95F.131 & MK440806 & 1119 & 372 & 41139.90 & 5.33 \\
\hline Sa99F.11 & MK440807 & 1113 & 370 & 40647.49 & 6.54 \\
\hline Sa9F.272 & MK440808 & 1920 & 639 & 61736.39 & 9.03 \\
\hline Sa9F.31 & MK440809 & 1164 & 387 & 42896.80 & 8.41 \\
\hline Sa9F.81 & MK440810 & 1557 & 518 & 53135.19 & 8.59 \\
\hline
\end{tabular}

3 


\section{Figure 1}

Phylogenetic relationships among the $S$. alfredii and Arabidopsis thaliana GELP families.

The tree was generated with ClustalW and MEGA 5.0 software using the Neighbor-joining method. SaGELPs are labeled in red, and AtGELPs are labeled in black. Different clades are represented by different colored backgrounds. Different sub-branches are represented by different colored branching lines. The pale blue solid circle represents leaf sorting. The size of circle corresponds to the bootstrap value. 


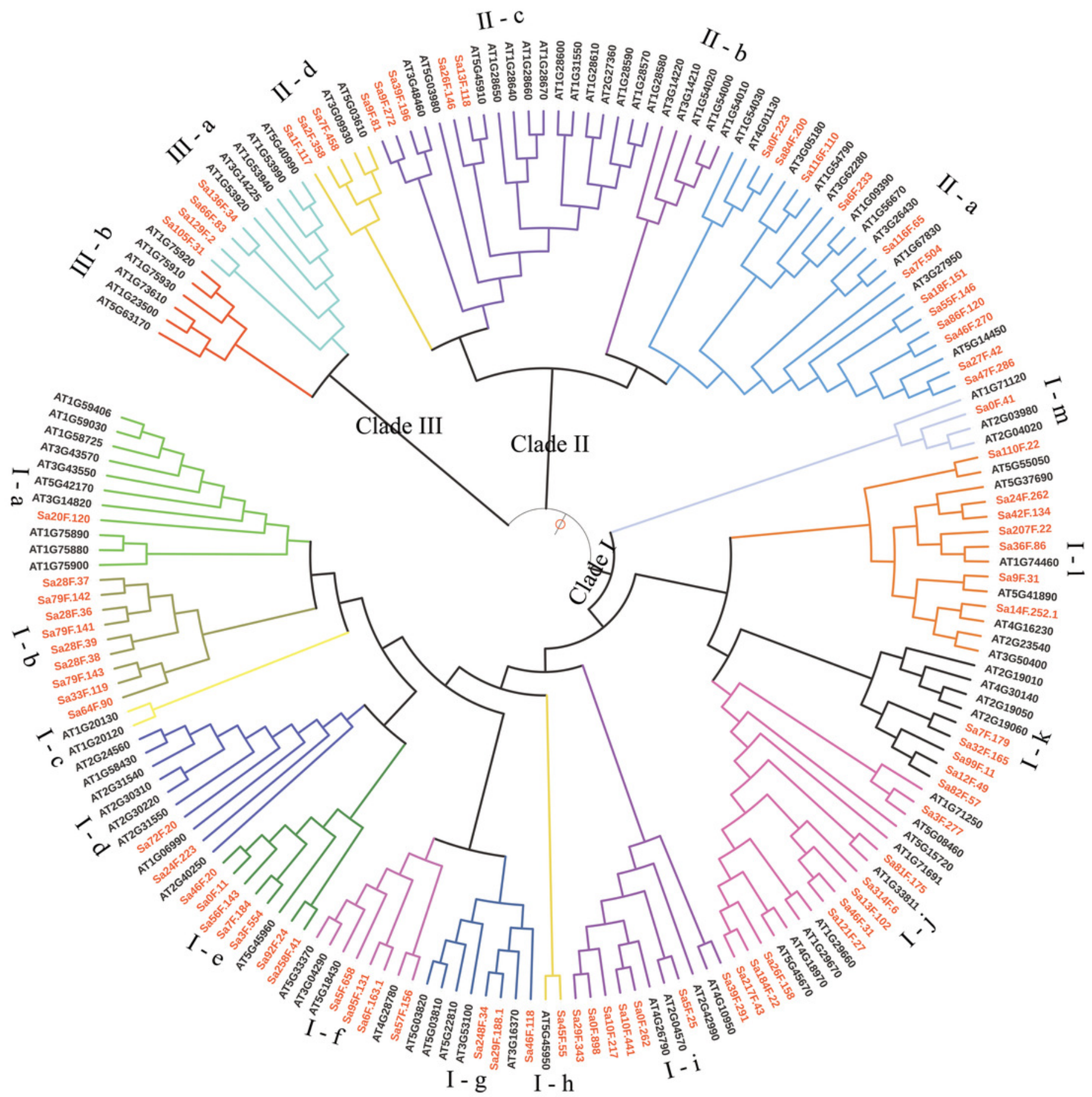




\section{Figure 2}

Gene structures and motif compositions of the GELP family in S. alfredii.

(A) The phylogenetic tree of SaGELP amino acid sequences was inferred using the Neighborjoining method and 1000 bootstrap replicates; (B) Gene structures of SaGELPs. Yellow boxes represent CDS, blue boxes represent upstream or downstream, lines represent introns; (C) Schematic representation of each of the conserved motifs in the SaGELPs selected by the MEME online tool. Different motifs are represented by different colored boxes. 


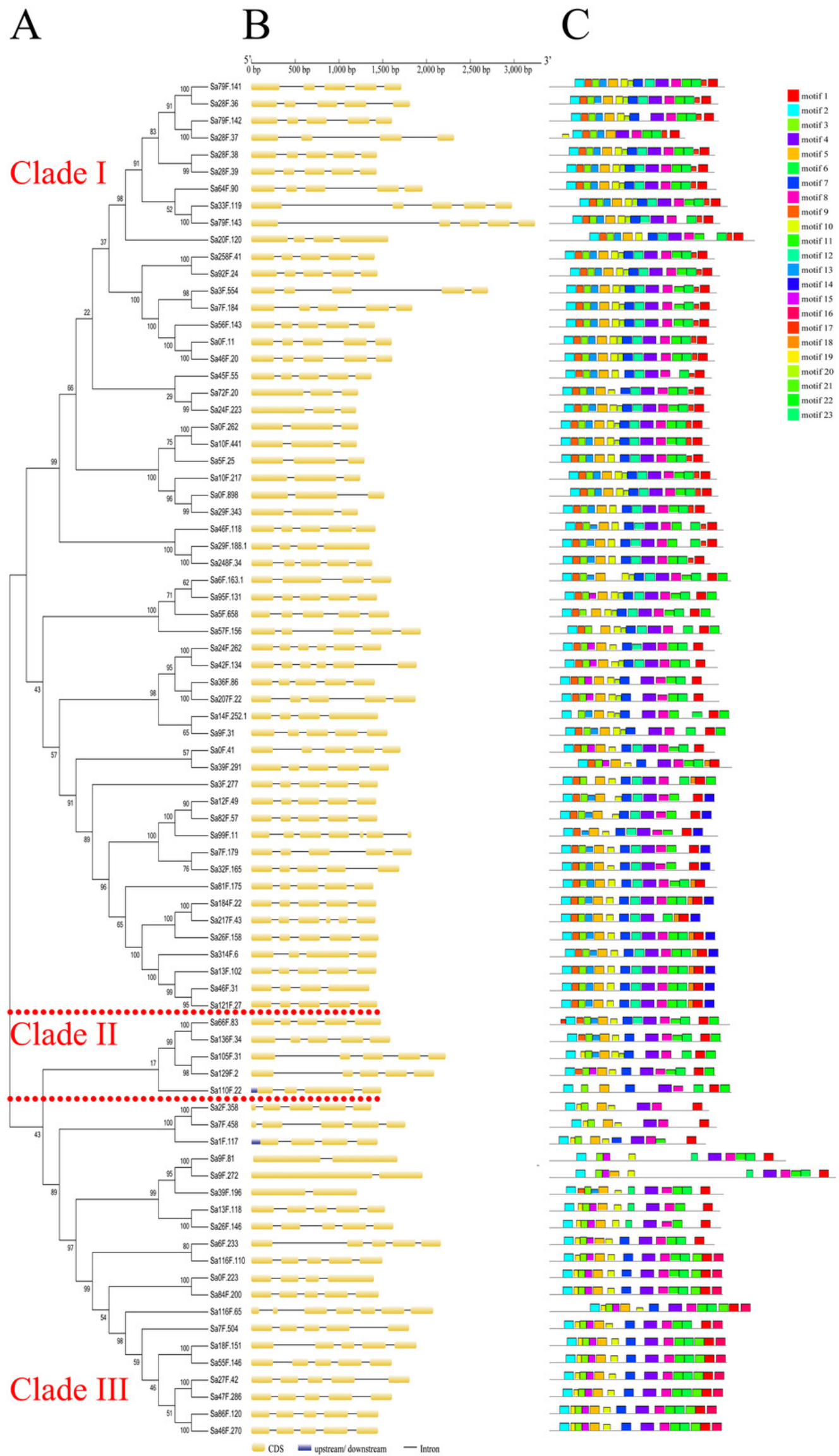


Figure 3

Four conserved motifs of $S$. alfredii GELPs.

The four consensus blocks I, II, III and V represent the conserved motifs in the amino acid sequences of the GDSL family, and are numbered based on their location from the $\mathrm{N}$ to $\mathrm{C}$ terminal. Triangles represent the conserved catalytic residues in conserved domains. Ser (S), Gly (G), Asn (N) and His (H) are conserved residues.
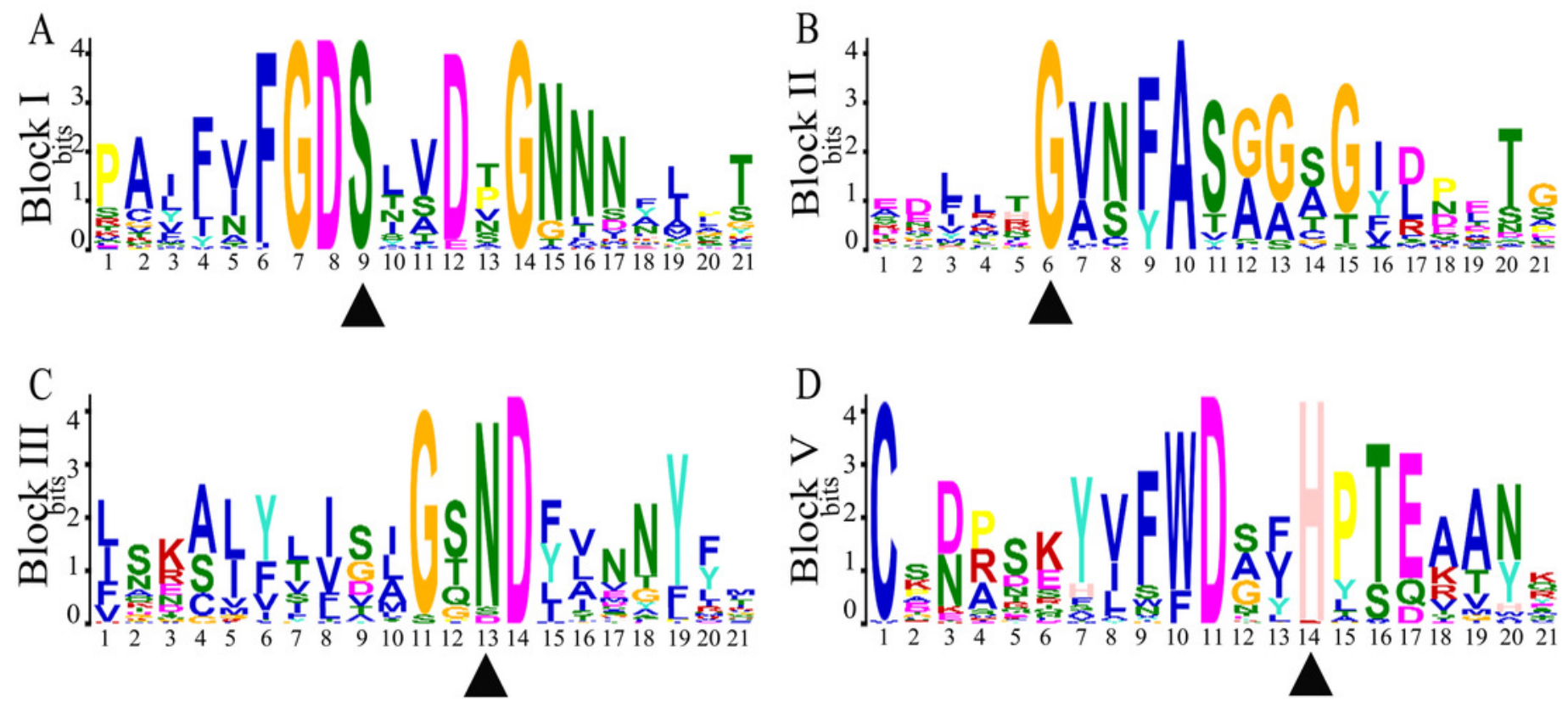
Figure 4

Cis-acting element analysis of promoters from the GELP family in S. alfredii.

Different elements are represented by different colored boxes. The box size corresponds to the element's sequence length. 
200

400

600

800

1000

1200

1400

map name

Saof.11

SaOF . 223 $1,1,1,1$

411114111

+

14, $1,11,1,1$

SaOF 41

SaOF. 898

Sa105F .31

Sa10F $4410 F$

Sa116F. 110

Sa116F. 65

Sa121F 27

Sa129F 2

Sa136F:34

$\mathrm{Sa} 13 \mathrm{~F} .102$

Sa $13 \mathrm{~F} \dot{1} 10$

Sa $14 \mathrm{~F} \cdot 252$.

Sa18F. 151

Sa207F 222
Sa20F 120

$\mathrm{Sa} 217 \mathrm{~F} .43$

Sa248F : 34

Sa24F. 262

$\mathrm{Sa} 58 \dot{F}^{2} 41$

Sa26F .146

Sa27F. 42

$\mathrm{Sa} 28 \mathrm{~F} \cdot 3$

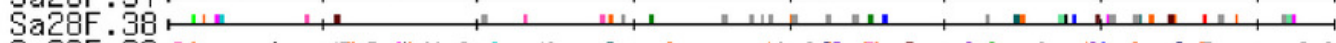

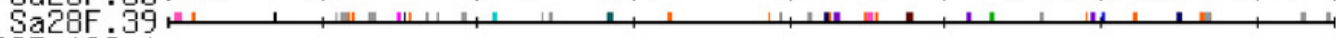

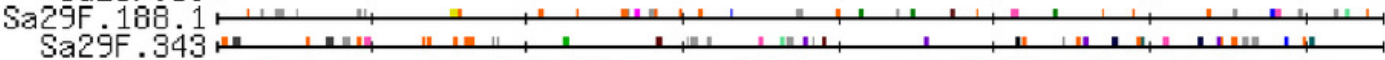

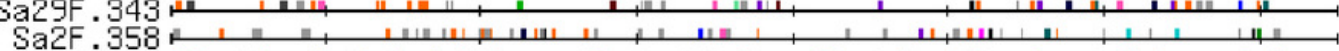

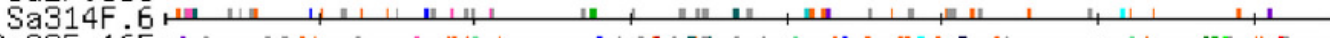

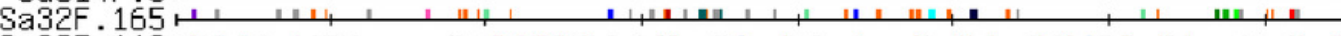

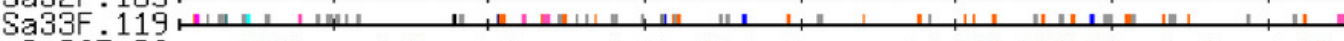

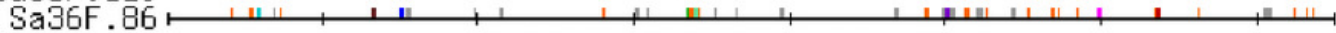

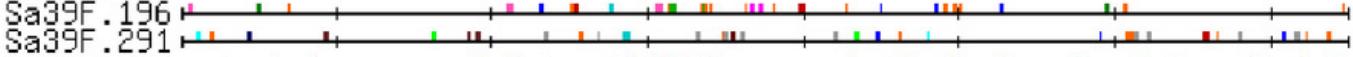

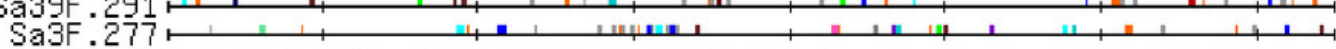

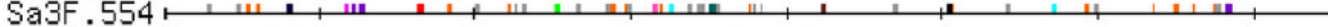

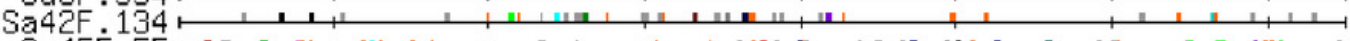

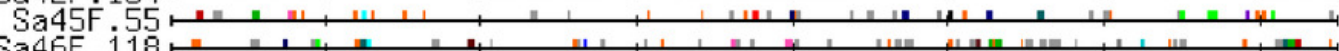

Sa46F $116 \longmapsto$

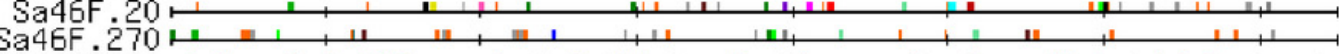

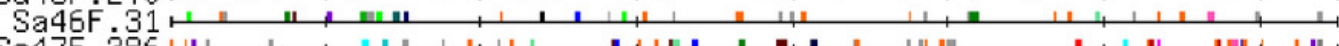

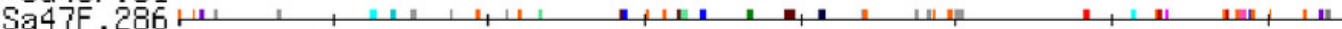

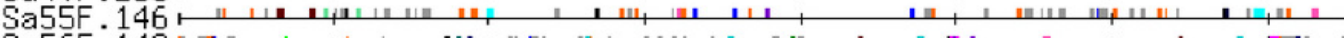

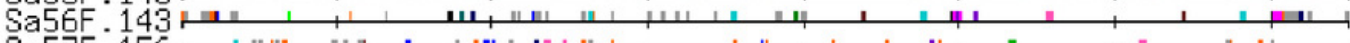

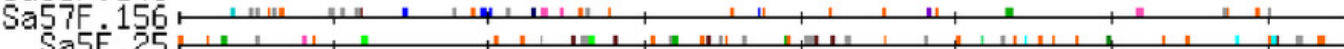

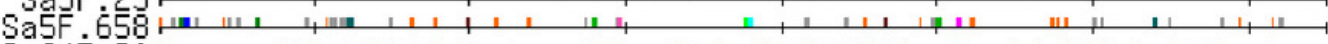

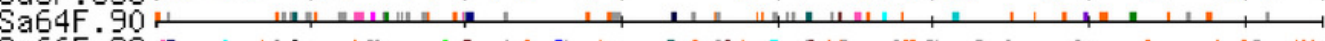

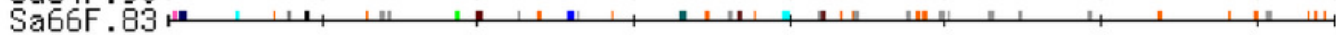

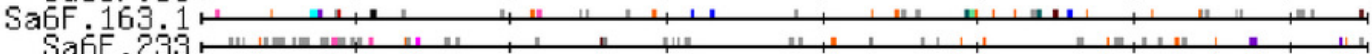

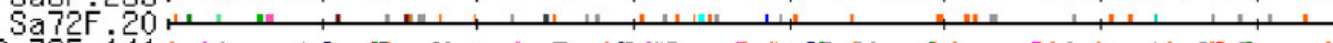

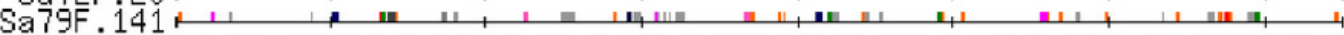

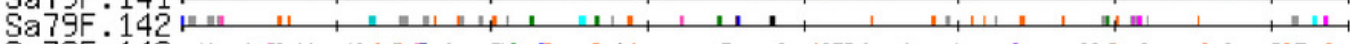

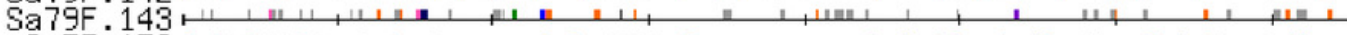

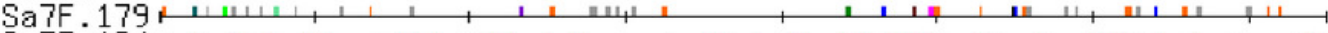

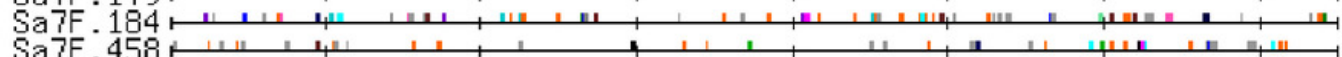

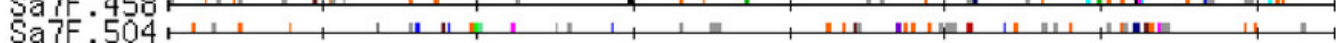

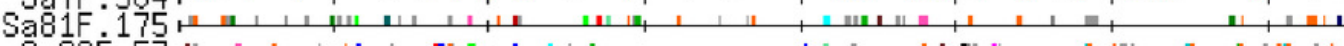

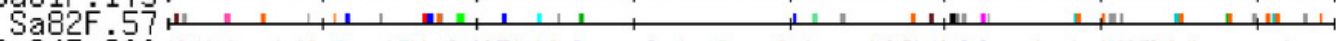

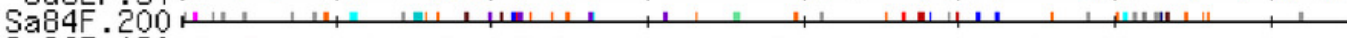

Sa86F 120 Sa92F

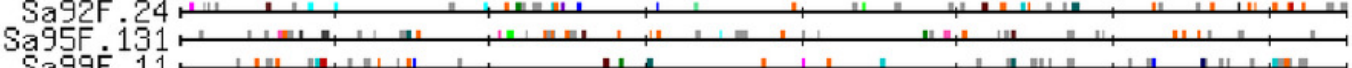

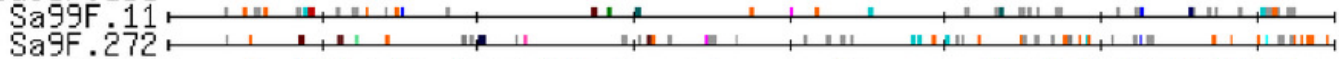

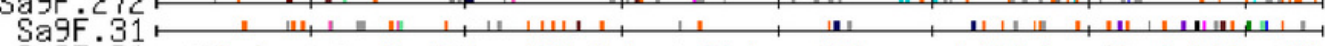

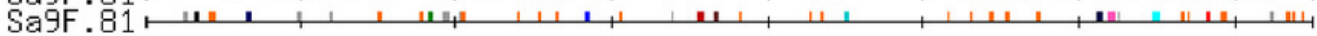

Legend

-identifier

ABRE

- $\mathrm{AE}-\mathrm{b}$ ox

$\operatorname{Box} 4$

Box I

I CAAT-box

- CAT-box

- CCAAT-box

- CCGTCC-box

- CGTCA-motif

CTAG-motif

IIRE

GAG-motif

I G-Bax

IIT1-motif

MBS

ITATA-box

- TCCACCT-motif

- AT-rich sequence

- AuxRR-core

- GATA-mot if

GCN4_motif

I-box

- LAMP-element

- Skn-1_motif

- TATC-box

TCA-element

IC-rich repeats

TGA-element

GARE-mot if

G-box

TGACG-motif

aRE

- CATT-mot if

HD-Zip 3

Sp1

IICT-motif

AT1-motif

Box II

- Gap-box

IIRE

- ATCT-motif

aT-rich element

HSE

ILTR

02-site

- ACA-motif

- ELI-box3

GA-motif

TCCC-motif

aTGCABAT motif

Box III

P-box

TATCCAT/C-motif

uUN-motif

Box-W1

a box

- $L-b o x$

MNF1

ACE

GCC box

MBSI

CE1

I $F-b a x$

SARE

E2Fb

- TGA-box

aTCC-motif

- GTGGC-mot if

as1

box $S$

- $c-b a x$

- GATT-mot if

E2F

- CG-motif

box $\mathrm{E}$

OBP-1 site

AACA_motif

1 GC-motif 
Figure 5

Co-expression network of S. alfredii GELP genes.

Nodes indicate genes, and edges indicate significant co-expression events between genes. Target genes involved in the same process are grouped together, and different groups are distinguished by different colors.

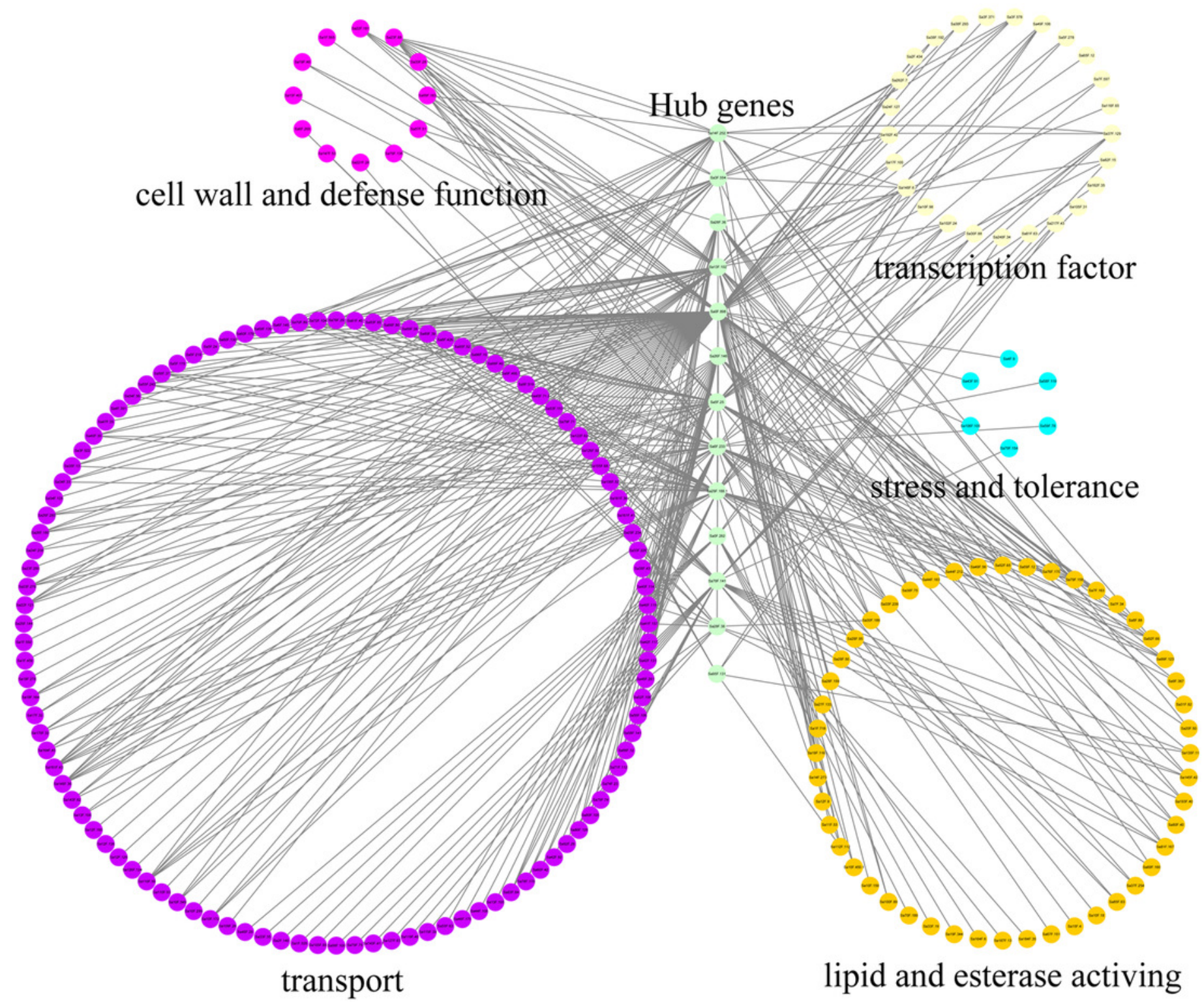




\section{Figure 6}

Expression profiles of $S$. alfredii GELPs in root (R), stem (S) and leaf (L) under normal and cadmium (Cd)-stress conditions.

The heat map shows the expression of 80 SaGELP genes. Each small square represents a gene, and its color represents the expression of the gene. The greater the expression, the darker the color (red, up-regulated; green, down-regulated). The "0" represents the control without $\mathrm{Cd}$ stress. The stress time were six hours (6 h) and seven days (7 d). 


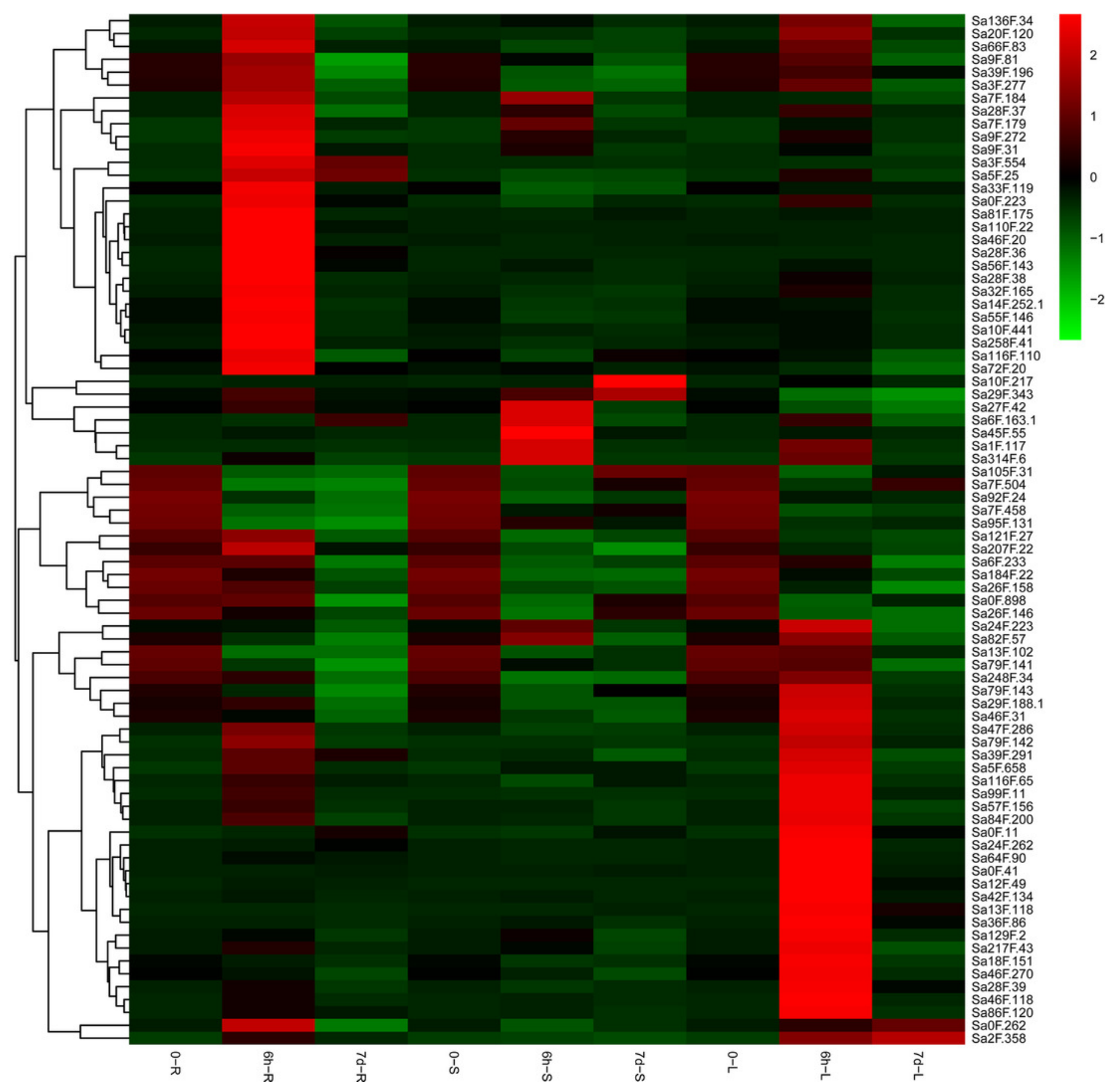




\section{Figure 7}

The expression profiles of $13 \mathrm{~S}$. alfredii GELP hub genes in different tissues under normal conditions.

(A) root; (B) stem; (C) leaf. The expression level of the control, Sa26F.146, (y-axis "Relative mRNA expression") was arbitrarily set to 1 . Bars indicate means \pm standard deviations (SDs) of at least three independent biological replicates. 

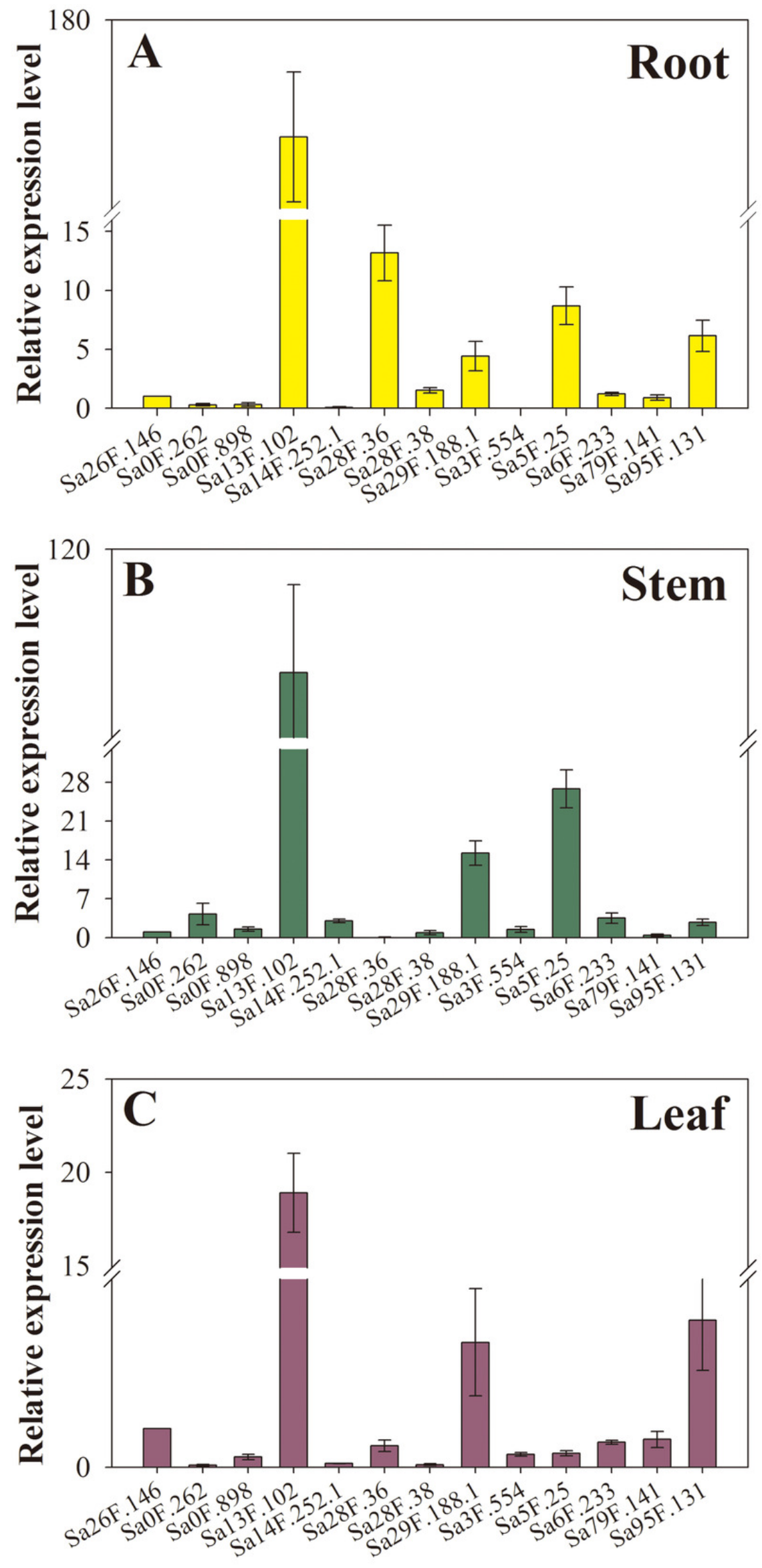

Peer) reviewing PDF | (2019:01:34381:1:1:NEW 6 Mar 2019) 


\section{Figure 8}

Overexpression of S. alfredii Hance GLIP8 increases the cadmium (Cd) tolerance and accumulation in yeast .

(A) the growth of $\triangle y c f 1$ yeast mutants transformed with the empty vector pYES2.0 or with pYES-DEST52 harboring SaGLIP8; (B) the accumulation of Cd in $\triangle y c f 1$ yeast cells. Bars indicate means \pm standard deviations (SDs) of at least three independent biological replicates. Two asterisks indicate a significant difference at $p<0.01$ compared with empty vector.

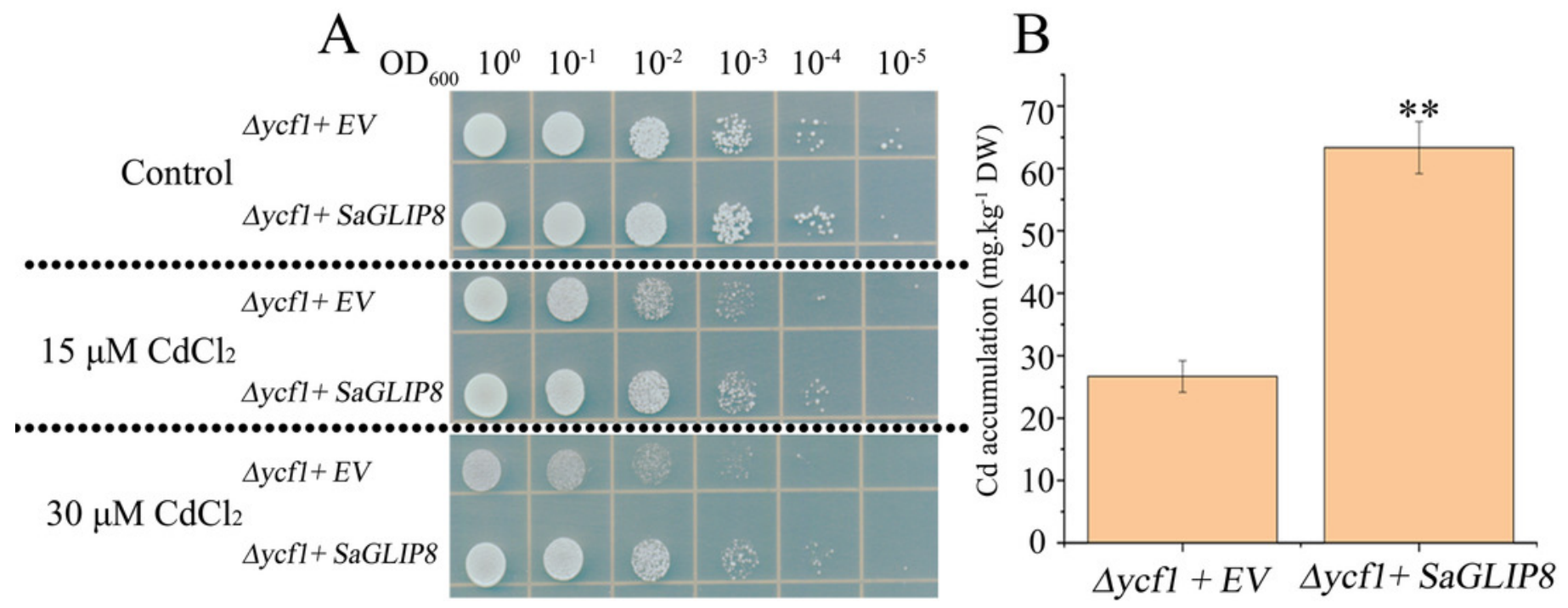

